\title{
Ci può essere a scuola l'assegno dei compiti?
}

\author{
Paolo D'Achille e Giuseppe Patota
}

PUBBLICATO: 14 GENNAIO 2020

\section{Quesito:}

Alcuni lettori ci chiedono se è corretto usare - come avviene in particolare in Campania - il nome maschile assegno nel significato di 'compiti assegnati per casa': "Devo fare l'assegno dei compiti" (o, semplicemente, "Devo fare l'assegno"); "La professoressa ha dato l'assegno dei compiti", "Scrivere l'assegno sul diario", e cosi via.

\section{Ci può essere a scuola l'assegno dei compiti?}

I 1 nome maschile assegno rappresenta un derivato per conversione dal verbo assegnare, che è la base anche di assegnamento e di assegnazione (in questo caso c'è però anche il precedente del latino assignationem). Rispetto a questi ultimi due termini, che hanno, oltre ad accezioni specifiche in diversi linguaggi settoriali, il significato generale di 'l'assegnare e il suo risultato', 'attribuzione', assegno nello standard è usato soltanto nel senso di 'somma assegnata a qualcuno' a vario titolo (assegno mensile, assegni familiari, assegno di ricerca, assegno vitalizio, ecc.) oppure come forma abbreviata di assegno bancario.

L'accezione di 'compiti assegnati per casa' è sconosciuta ai molti lessicografi che hanno redatto o diretto i tanti vocabolari che raccolgono e descrivono il lessico dell'italiano contemporaneo: non c'è traccia di questa accezione nel GRADIT Grande dizionario italiano dell'uso UTET e neppure nel Vocabolario Treccani 2008, nel Sabatini-Coletti 2008, nel Devoto-Oli 20I9, nel Garzanti 20I7 e nello Zingarelli 20I9.

La documentazione offerta dalla rete (in interventi in cui si affronta la questione dell'opportunità o meno di assegnare compiti a casa agli scolari) sembra confermare la diffusione di assegno in senso "scolastico" prevalentemente in Campania. Alcuni esempi recenti, tuttavia, compaiono in testi non sospettabili di interferenze regionali:

Più compiti a casa non significa necessariamente una migliore istruzione. Secondo l'inchiesta dell'Ocse maggiore è l'assegno minore è il rendimento. (frase documentata il 27 dicembre 2016 in vari siti: www.disal.it, https://libreriamo.it)

Un assegno che non sia stato iscritto nel registro di classe sarebbe teoricamente nullo, legittimandovi a opporre al professore la mancata segnalazione dei compiti sul registro. (Alessandro Cappuccio, Il prof ci assegna i compiti via Whatsapp: è legale?, 20/04/2015, in cui però compare più spesso assegnazione)

Quando si demanda all'alunno ed alla mamma-papà pezzi di apprendimento, su cui non si è già lavorato e non ci si è esercitati prima in classe, vuol dire che siamo di fronte ad una scuola che non risponde adeguatamente al suo compito, che la sua azione è incompleta ed ingiusta, che dichiara di essere scuola di tutti e di ciascuno, operativa e laboratoriale, ma poi nei fatti è ancora appesa alla vecchia triade dei tempi passati, statica e rigida: verifica-interrogazione sui compiti fatti a casa e, nel poco tempo che rimane, spiegazione frontale e, in fretta, l' "assegno" dei nuovi compiti da fare a casa. (Domenico Sarracino, Compiti si, compiti no o compiti come?, I8/ro/20I6; si notino però le virgolette). 
Un esempio più antico, reperito grazie a Google Libri, si trova nel periodico "La nuova scuola italiana. Rivista magistrale settimanale" del I933, in un articolo intitolato "I compiti per casa", in cui si legge:

Per certi maestri non è Natale se non fanno un lungo assegno di compiti. Lasciamo che le feste siano godute in pace!

Ma l'articolo è firmato Parthènope e sia questo dato, sia la diffusione prevalentemente campana dei cognomi degli autori degli articoli più recenti di cui abbiamo riportato i passi (Cappuccio e Sarracino) sembrano riportare all'area indicata dai nostri lettori. Tuttavia di un "assegno dei compiti a casa" si parla già nel periodico "I diritti della scuola" del igo6, edito a Roma.

Cosi stando le cose, dobbiamo concludere che il significato "scolastico" della parola assegno è nato in ambito burocratico - dove del resto formazioni del genere sono tutt'altro che rare (allaccio, affido, ecc.) - e poi è rimasto circoscritto in ambito regionale (specificamente in Campania, almeno per quanto ne sappiamo), dove ha assunto il significato di 'complesso dei compiti assegnati', che (almeno finora) non è entrato nell'italiano comune. Sconsigliamo, dunque, di farne uso.

\section{Cita come:}

Paolo D'Achille e Giuseppe Patota, Ci può essere a scuola l'assegno dei compiti? , "Italiano digitale", 2020, XII, 2020/1 (gennaio-marzo)

DOI: $10.35948 / 2532-9006 / 2020.3316$

Copyright 2020 Accademia della Crusca

Pubblicato con licenza creative commons CC BY-NC-ND 